\title{
Analysis of Walking Accessibility of Park Green Space in Weidu District of Xuchang City Based on GIS
}

\author{
Yage Cui ${ }^{1}$, Wenhao Tan ${ }^{1}$, Linli Cheng ${ }^{1}$, Tingting Chen ${ }^{1}$, Liang Lv ${ }^{1}$ and Hui Pan ${ }^{1,2 *}$ \\ ${ }^{1}$ College of Landscape Architecture, Fujian Agriculture and Forestry University, Fuzhou, Fujian 350002, China \\ ${ }^{2}$ Department of Tourism, Minjiang University, Fuzhou, Fujian 350108, China
}

\begin{abstract}
Taking the park green space in Weidu District of Xuchang City as the research object, the spatial service area of the park green space under the mode of pedestrian transportation is analyzed by using the grid analysis function of geographic information system (GIS). The results show that under the walking mode in the research area, only less than $1 / 10$ of the residents can walk to the park within 10 minutes, and nearly $1 / 5$ of the residents can enjoy the service function of urban park green space within 20 minutes. Under the walking mode of 30 minutes, the accessibility area of park green space is only $36.63 \%$, which is mainly concentrated in the new urban area. There are many urban parks with large scale. The number of urban parks in the old urban area is small and the accessibility is relatively poor. The research results can provide theoretical basis for optimizing the spatial structure of green space in Weidu District of Xuchang City.
\end{abstract}

\section{Introduction}

Urban green space is an important part of urban modernization and an important green infrastructure. It not only provides a beautiful natural environment for the residents, but also has the functions of protecting the environment, improving the ecology, purifying the water body and blocking the noise. At the same time, it also has the functions of improving the city style, preventing and avoiding disasters, storing water and flood control, alleviating the effect of urban heat island and improving the life quality of the residents. In addition, as a public infrastructure, the park green space also provides a place for citizens to have leisure and entertainment activities. It has the functions of popular science, publicity, education and many other public services. It is an important part of residents' life and one of the bases for judging residents' quality of life and improving residents' happiness index. With the improvement of residents' living standards, as well as the enhancement of natural awareness and human rights awareness, residents' demand for urban parks with natural environment as the main carrier increases, and their focus also changes from the level of pure natural environment quality to the high level of park comprehensive service ability, and the accessibility of public parks is an important standard to measure the park comprehensive service ability ${ }^{[1]}$. The urban development of Xuchang City starts late, and the process is relatively slow. Due to the limitation of natural geographical conditions and the lack of scientific urban planning in the early stage, the urban development is faced with the problems of less overall distribution of green infrastructure, less park green space, and unreasonable spatial distribution. Therefore, it is necessary to analyze the accessibility of urban park green space in Xuchang City, find out the problems existing in the planning and put forward countermeasures, so as to provide scientific guidance for the planning and construction of park green space in Xuchang City in the later stage.

Hansen first proposed the concept of accessibility, which means the size of interaction opportunities between nodes in the traffic network ${ }^{[2]}$. The commonly used measurement methods of accessibility mainly include buffer analysis, cost distance method, network analysis method ${ }^{[3]}$. At present, the main research focuses on the accessibility of urban park green space, the fairness of landscape and the optimization of landscape spatial pattern. In China, Yu Kongjian first evaluated the service function of urban green space in Zhongshan City with landscape accessibility as the analysis index ${ }^{[4]}$. Han Zhigang et al. studied the spatial pattern of multi-scale accessibility in Henan Province based on cost distance ${ }^{[5]}$. Luo Miaomiao et al. conducted a study on the evaluation of park green space in the central urban area of Tianjin City from the perspective of spatial justice ${ }^{[6]}$. Chen Feng conducted the accessibility analysis and layout optimization research of rural cultural facilities based on GIS network analysis method ${ }^{[7]}$. Especially with the maturity of geographic information technology and the further application of $3 \mathrm{~S}$ technology in the landscape industry, the research on the accessibility of park green space is gradually in-depth, the perspective of research is more abundant, and many excellent achievements have emerged.

\footnotetext{
* Corresponding author: 332088289@qq.com
} 
The accessibility of urban park is defined as the difficulty level of overcoming space resistance in the process of residents' arriving at the park from their place of residence. Influenced by time, distance, cost and other factors, it can objectively reflect the rationality of the spatial layout of urban park green space, and it is an important index to evaluate whether residents can enjoy urban green space fairly and conveniently ${ }^{[8]}$.

In this study, Urban Park Green Space System of Weidu District of Xuchang City is taken as the research object, the database based on urban road system and urban park green space is established by using GIS grid analysis function, the problems existing in the accessibility of urban park green space under three different time modes of walking $10 \mathrm{~min}, 20 \mathrm{~min}$ and $30 \mathrm{~min}$ are analyzed, and the optimization strategy is put forward.

\section{Description of Research Area}

Xuchang City is located in the middle of Henan Province, east longitude $113^{\circ} 03^{\prime} \sim 114^{\circ} 19^{\prime}$, north latitude $33^{\circ} 42^{\prime} \sim 34^{\circ} 24^{\prime}$. It borders on Zhoukou, Luohe, Pingdingshan, Zhengzhou and Kaifeng with a total area of 4,996 square kilometers. In the study, Weidu District of Xuchang City Center is taken as the research object, and the areas within G107 and S220 expressway are taken as the research area, of which the urban park area is 391.614 hectares, accounting for $2.44 \%$ of the total research area.

\section{Research Method}

\subsection{Data Source}

Download the OSM map data of Xuchang City in OpenStreetMap (referred to as OSM for short), analyze and process the data through the LoadOSMFile tool under OpenStreetMapToolbox module in ArcGis10.2, and extract the data information such as main road, secondary road, branch road, urban park green space and its main entrance location according to the research needs (see Fig 1).

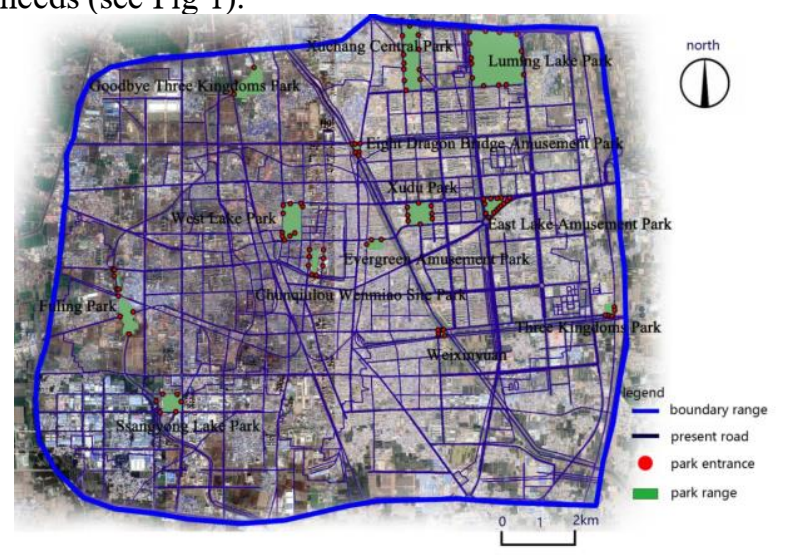

Fig. 1. Distribution map of urban road and park entrance

\subsection{Research Method}

\subsubsection{Network Analysis Method}

Geographic network is a reticular formation composed of several lines and points. Network resources flow along this linear network. The fundamental elements of a network are linear entities and the intersection points of these linear entities, also known as chains or network lines and nodes. A complete network includes center, chain, node and resistance (see Fig 2), and center is the main research object. In this study, the node is the crossroads, the chain is the traffic route from residents to the park, the center is the entrance and exit of each park, and the resistance is the speed and node delay of different traffic modes ${ }^{[9]}$.

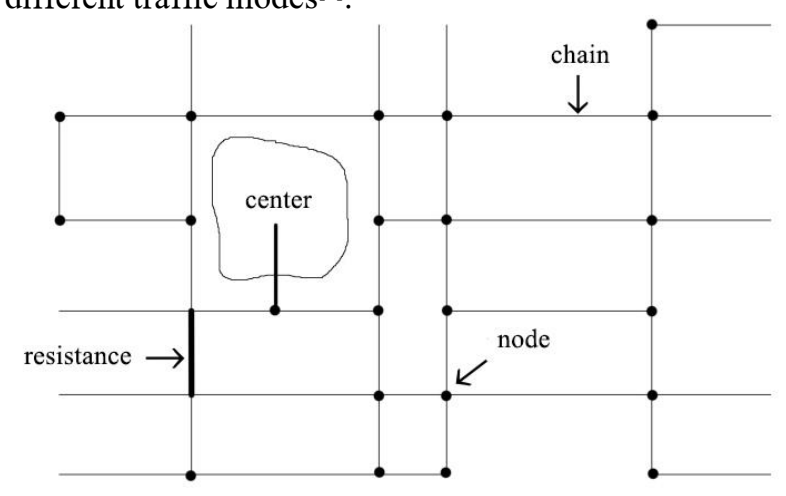

Fig. 2. Fundamental elements of network model

\subsubsection{Build a Network Data Set}

Build a network data set according to vectorization road screened well. First, add 'shapefile' file of park entrance and vectorization road; second, give fundamental field attributes required by road network, that is, road length, level and time attributes; finally, set network connectivity, specify network data set attributes and set resistance value to build a network data set. In today's cities, general roads can run in two directions, but some roads can only run in two directions in a fixed period of time. According to the actual situation of Xuchang City, all roads are allowed to pass in two directions in this study.

\subsubsection{Accessibility Analysis of Green Space}

The service scope of urban park green space refers to the area that can be reached within a specific distance or time starting from the urban park green space. When calculating the service scope of urban park green space, it is necessary to consider the travel mode, consumption time, psychological expectation time, and urban road level and its characteristics ${ }^{[10]}$. This study is conducted mainly on walking, a way of travelling for residents, and the accessibility index is time spending on reaching the urban park green space in $0 \sim 10 \mathrm{~min}, 10 \sim 20 \mathrm{~min}$ and 20 30min, which is evaluated by good, general and poor respectively. The network Analyst module in ArcMap10.2 is used to build the analysis layer of service area, set the impedance of 10min, 10 20min and 
20 30min, and generate the service range of urban park green space within three time-indexes.

\section{Analysis of Walking Accessibility of Green Space Based on GIS}

\subsection{Analysis on the Overall Accessibility of Urban Park Green Space under Walking Mode}

The overall accessibility of park green space is an important index to measure the service capacity of urban park green space. In this paper, the network text analysis method is selected to evaluate the overall accessibility of the park green space. Walking to the park is the most common way for people to travel after a meal, and it is the highest embodiment of the accessibility of urban park green space. Compared with other travel modes, walking to the park is the most convenient, which can best reflect the fairness of urban park green space ${ }^{[1]}$. The General Environmental Administration of Europe once suggested that the walking time of common urban park green space should be within 15 minutes, and the accessibility of walking way, to a large extent, reflects the development degree of current urban road traffic and the layout rationality of urban park green space. The difference of residents' age structure should be considered when walking, and the travel speed is different. According to different travel speeds of children, young people, middle-aged people and the elderly, the average walking speed of $60 \mathrm{~m} / \mathrm{min}$ is selected in the study. According to the walking speed attribute and road distance, the road time attribute is calculated and given, i.e. time resistance value ${ }^{[13]}$. Because the road hierarchy has little influence on the walking speed of residents, this study does not consider the influence of road hierarchy on the accessibility of urban park green space. Through the calculation of ArGIS service scope, the walking accessibility regional distribution map of urban park green space in Xuchang City within different time of 10min, 10 20min and 20 30min (as shown in Fig 3) is obtained. In order to display the walking accessibility of urban park green space more intuitively and conveniently, the regional area and proportion of different travel time are summarized through ArGIS, and the results are shown in Table 1.

It can be seen from table 1 that under the walking mode, the service area with good accessibility (consumption time $0 \sim 10 \mathrm{~min}$ ) is $1350.45 \mathrm{~km}^{2}$, only accounting for $8.417 \%$ of the total area of the research area; the service area with general accessibility (consumption time $10 \sim 20 \mathrm{~min}$ ) is $3432.30 \mathrm{~km}^{2}$, accounting for $21.392 \%$ of the total area of the research area; the service area with poor accessibility (consumption time 20 30min) is $5877.29 \mathrm{~km}^{2}$, accounting for $36.630 \%$ of the total area of the research area. Under the comprehensive walking mode, the total service area of the accessibility of urban park green space is $10660.04 \mathrm{~km}^{2}$, accounting for $66.439 \%$ of the total area of the research area. It can be seen from the calculation results that in the research area of Xuchang City, more than $1 / 3$ of the residents cannot use the function of urban park green space by walking within 30 minutes. It can be seen that the walking accessibility of park green space in Weidu District of Xuchang City is generally poor.

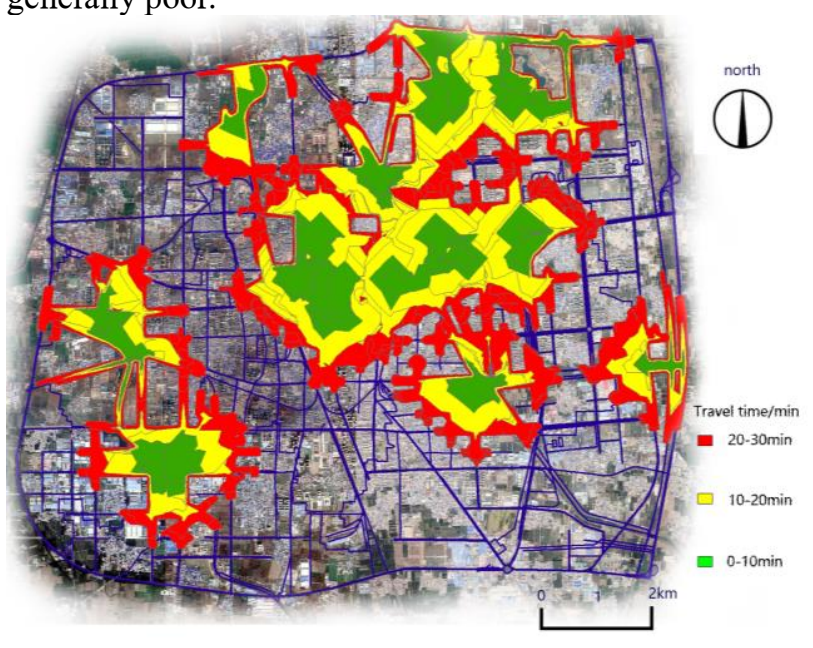

Fig 3 Analysis graph of walking accessibility of urban park green space

Table 1. Accessibility scope of urban park green space under fixedtime walking

\begin{tabular}{|c|c|c|}
\hline Travel time/min & $\begin{array}{c}\text { Covered } \\
\text { area/km² }\end{array}$ & $\begin{array}{c}\text { Percentage of } \\
\text { study area/\% }\end{array}$ \\
\hline $\begin{array}{c}\text { 0-10 (Good } \\
\text { accessibility) }\end{array}$ & 1350.45 & 8.417 \\
\hline $\begin{array}{c}\text { 10-20 (Average } \\
\text { reachability) }\end{array}$ & 3432.30 & 21.392 \\
\hline $\begin{array}{c}\text { 20-30 (Poor } \\
\text { accessibility) }\end{array}$ & 5877.29 & 36.630 \\
\hline statistics & 10660.04 & 66.439 \\
\hline
\end{tabular}

\subsection{Analysis on the Overall Layout of Park Green Space}

Observing Fig 4, it can be found that the service area of urban park green space is expanding within $10 \mathrm{~min}, 10$ $20 \mathrm{~min}$ and $20-30 \mathrm{~min}$, indicating that the overall accessibility of park green space is increasing with the extension of walking time. It can also be seen from the figure that even part of the central urban area with centralized Park distribution is not within the park service scope, which indicates that the overall spatial distribution of urban parks in Xuchang City is unreasonable, the park service scope is generally distributed in dots without forming a region, and the service area is mainly concentrated in the middle and northeast of the research area, and there are relatively few branches in the southwest of the city, even without park green space. At present, there are great defects in the layout rationality of urban park green space.

The service area of urban park green space is distributed in dots within 10 minutes of walking, and the 
service scope only accounts for $8.417 \%$ of the total area of the research area. The parks are not connected with each other, and there is no overlap between the areas, which indicates that less than $10 \%$ of the residents can enjoy the urban green space within 10 minutes of walking, and the residents have little choice to visit the urban park. In addition, nearly $91.583 \%$ of urban residents can not enjoy the park green space within 10 minutes of walking, and the accessibility of park green space is poor as a whole. The urban park that within 10 to 20 minutes of walking can reach an area of 3432.30 hectares, accounting for $21.392 \%$ of the total area of the research area. The service scope of park green space is wider. The park service scope of Luming Lake Park, Central Park, Balongqiao Park, Xudu Park, East Lake Park, Changqing Park, West Lake Park and the Spring and Autumn Tower Confucian Temple Heritage Park is connected into a piece. The service scope overlaps with each other, and urban public park group also appears. These show that under the condition of walking for 20 minutes, the service scope of urban park green space is wider, the overall accessibility is enhanced, even some regional residents can reach multiple parks within 20 minutes, and the residents have greater travel options. Under the condition of walking for 20-30 minutes, the service area of urban park green space reaches 5877.29 hectares, accounting for $36.630 \%$ of the total area of the research area. At this time, most of the park service areas have been connected into pieces, and the group phenomenon is more obvious. Only the Three Kingdoms Park Expo Park is independent, which may be related to the public park's proximity to the suburbs and inconvenient transportation. These show that the overall accessibility of urban parks is significantly enhanced and the service scope is wider when walking for 30 minutes.

\section{Conclusions and Suggestions}

\subsection{Conclusions}

In this paper, the accessibility of urban park green space in Xuchang City is analyzed based on different transportation modes, and the conclusions are as follows:

(1) Under the walking mode, the accessibility service scope of urban park green space is mainly concentrated in the new urban area, while the old urban park is less distributed and covers a relatively small area; In addition, due to the inconvenient transportation in the old urban area, the overall accessibility of individual park green space is poor, and the accessibility of space is not good. Moreover, with the increase of the number of residents in the high-tech zone and the development zone, the contradiction that the existing urban green space cannot meet the needs of urban residents will become more and more prominent.

(2) The existing urban park green space layout is unreasonable, and the park location is relatively concentrated. Large urban parks are mainly distributed in the middle and north of Weidu District, and the southern old urban area with relatively concentrated population basically has no park coverage; At present, the urban construction of Xuchang City is gradually expanding outward, and the number of urban residents in the Northeast development zone and high-tech zone is increasing day by day. The current urban park green space cannot meet the needs of the whole urban residents.

(3) The walking accessibility of park green space in Weidu District of Xuchang City is generally good, but the overall accessibility of individual park green space is very poor; With the walking time longer, the overall accessibility of park green space is increasing.

(4) The existing urban park green space in Weidu District of Xuchang City is distributed in scattering, with poor overall cohesion. There is no organic complementary whole between the parks, and the urban green infrastructure is inadequate, so it is difficult to play the maximum ecological value.

\subsection{Suggestions}

(1) The poor accessibility of urban parks in the old urban area is related to the lack of urban planning in the early stage, the imperfection of urban road system and the unreasonable allocation of land resources. In addition, the old urban area is densely populated, densely built, with short land use, less urban park planning, small area and fragmented for each other. Moreover, with the relatively partial position, belonging to old urban area, low road quality and level, and the poor traffic capacity, all of this result in poor overall accessibility of the park. In this regard, we should strengthen the road planning and construction, improve the road system of the old urban area, accelerate the demolition and reconstruction of the old urban area, promote the land use renewal, increase the number of parks in the old urban area, plant trees wherever there is space, so as to improve the accessibility of the park green space. And combine the demand of urban residents for green space with road level and traffic mode to reduce the travel resistance of residents, so as to make scientific and reasonable guidance for the later planning and spatial layout of urban green space system in Xuchang City.

(2) Reasonably plan and arrange the park distribution of the development zone and the high-tech zone, plan and integrate the original urban park green space, establish the perfect urban park system, improve the urban green infrastructure, and improve the service scope of the urban park green space.

(3) Establish travel mechanism under different traffic modes to ensure the independent operation of each traffic system. On the one hand, it reduces the travel time cost of residents, increases safety and convenience. On the other hand, it improves the accessibility of urban park green space system.

(4) The macro planning of the city incorporates the construction of urban parks, connect with the land use planning and the overall planning of the city. Do the toplevel design well. Guide the construction of urban parks through the top-level planning, improve the green infrastructure of the city, give full play to the ecological 
function of the green space of urban parks, and improve the coverage and accessibility of urban parks.

This paper only considers the time cost of walking, which is a single mode of transportation, and does not consider the impact of riding, car, bus exchange, transfer and space resistance in different periods. These factors will also affect the accessibility evaluation of urban park green space.

\section{References}

1. Xie Huanjing, Liang Ping, Shen Qinwei, Xiong Huijin, Lin Jiaojiao, Chen Xiaoyun, Lu Dongfang, Zheng Yushan. Landscape Pattern and Accessibility Analysis of Taiyuan City Based on GIS [J]. Journal of Southwest University (Natural Science Edition), 2019, 41 (11): 132-140.

2. HANSEN W G.How accessibility shapes land use[J].Journal of the American Institute of Planners, 1959,25: 73- 76.

3. Li Bo, Song Yun, Yu Kongjian. Evaluation method of accessibility index in urban park green space planning [J]. Journal of Peking University (Natural Science Edition), 2008 (04): 618-624.

4. Yu Kongjian, Duan Tiewu, Li Dihua, Peng Jinfu. Evaluation methods and cases of landscape accessibility as an index to measure the function of urban green space systems [J]. Urban Planning, 1999 (08): 7-10 + $42+63$.

5. Han Zhigang, Cui Caihui, Miao Changhong, Wang $\mathrm{Xi}$, Chen Changyou. Spatial pattern of multi-scale reachability in Henan Province based on cost distance [J]. Journal of Henan University

6. Luo Miaomiao. Research on the Evaluation of Green Space in Downtown Parks of Tianjin City from the Perspective of Spatial Justice [D]. Tianjin University of Commerce, 2019. (Natural Science Edition), 2019, 49 (02): 127-136.

7. Chen Feng. Accessibility analysis and layout optimization of rural cultural facilities based on GIS network analysis [D]. Chang'an University, 2019.

8. Zhai Xue, Zhang Tao, Wang Ze. Study on the accessibility of green space in mountainous urban parks based on network analysis method: A case study of Chengde downtown [J]. Forestry and Ecological Sciences, 2018,33 (03): 336 -342.

9. Xie Huanjing, Shen Qinwei, Wei Lingwei, Zheng Yushan, Lu Dongfang. GIS-based accessibility analysis of Liaocheng Park green space [J]. Journal of Henan University of Science \& Technology (Natural Science Edition), 2019, 47 (01): 31-36.

10. Fang Jiaxuan. Evaluation of pedestrian accessibility in Nanshan District of Shenzhen based on POI [D]. Harbin Institute of Technology, 2017.

11. Chen Shuqian. Study on the accessibility of park green space based on network analysis [D]. Harbin Institute of Technology, 2013. 